\title{
Task scheduling and resource allocation in cloud computing using a heuristic approach
}

\author{
Mahendra Bhatu Gawali ${ }^{{ }^{*}}$ and Subhash K. Shinde ${ }^{2}$
}

\begin{abstract}
Cloud computing is required by modern technology. Task scheduling and resource allocation are important aspects of cloud computing. This paper proposes a heuristic approach that combines the modified analytic hierarchy process (MAHP), bandwidth aware divisible scheduling (BATS) + BAR optimization, longest expected processing time preemption (LEPT), and divide-and-conquer methods to perform task scheduling and resource allocation. In this approach, each task is processed before its actual allocation to cloud resources using a MAHP process. The resources are allocated using the combined BATS + BAR optimization method, which considers the bandwidth and load of the cloud resources as constraints. In addition, the proposed system preempts resource intensive tasks using LEPT preemption. The divide-and-conquer approach improves the proposed system, as is proven experimentally through comparison with the existing BATS and improved differential evolution algorithm (IDEA) frameworks when turnaround time and response time are used as performance metrics.
\end{abstract}

Keywords: Cloud computing, Task scheduling, Heuristic, Resource management, Analytic hierarchy system, BATS, BAR

\section{Introduction}

Cloud computing is an accelerating technology in the field of distributed computing. Cloud computing can be used in applications that include storing data, data analytics and IoT applications [1]. Cloud computing is a technology that has changed traditional ways in which services are deployed by enterprises or individuals. It provides different types of services to registered users as web services so that the users do not need to invest in computing infrastructure. Cloud computing provides services such as IaaS (Infrastructure as a Service), PaaS (Platform as a Service), and SaaS (Software as a Service) [2]. In each type of service, the users are expected to submit the requests to the service provider through the medium of the Internet. The service provider is responsible for managing the resources to fulfill the requests generated by users. Service Providers employ scheduling algorithms to schedule the incoming request (tasks) and

\footnotetext{
* Correspondence: gawali.mahen@gmail.com

${ }^{1}$ Department of Information Technology, Thadomal Shahani Engineering

College, Bandra (W), University of Mumbai, Mumbai, MS, India

Full list of author information is available at the end of the article
}

to manage their computing resources efficiently. Task scheduling and resource management permit providers to maximize revenue and the utilization of resources up to their limits. In practice, in terms of the performance of cloud computing resources, the scheduling and allocation of resources are important hurdles. For this reason, researchers have been attracted to studies of task scheduling in cloud computing. Task scheduling is the process of arranging incoming requests (tasks) in a certain manner so that the available resources will be properly utilized. Because cloud computing is the technology that delivers services through the medium of the Internet, service users must submit their requests online. Because each service has a number of users, a number of requests (tasks) may be generated at a time. Systems that do not employ scheduling may feature longer waiting periods for tasks moreover, some short-term tasks may terminate, due to the waiting period. At the time of scheduling, the scheduler needs to consider a number of constraints, including the nature of the task, the size of the task, the task execution time, the availability of resources, the task queue, and the load on the 
resources. Task scheduling is one of the core issues in cloud computing. Proper task scheduling may result in the efficient utilization of resources. The major advantage of cloud computing is that it promotes proper utilization of resources [3]. Thus, task scheduling and resource allocation are two sides of a single coin. Each affects the other.

Currently, Internet users can access content anywhere and anytime, without needing to consider the hosting infrastructure. Such hosting infrastructure consists of various machines with various capabilities that are maintained and managed by the service provider. Cloud computing enhances the capabilities of such infrastructure, which can access the Internet. Cloud service providers earn profits by providing services to cloud service users.

The cloud service end user can use the entire stack of computing services, which ranges from hardware to applications. Services in cloud computing employ a pay-as-you-go basis. The cloud service end user can reduce or increase the available resources, per the demands of the applications. This is one the major advantages of cloud computing, but service users may be responsible for paying additional costs for this advantage. The cloud service user can rent the resources at any time and release them with no difficulty. The cloud service user has the freedom to employ any service based on application need. The freedom of service choice for users has led to problems; that is the next user request cannot be perfectly predicted. Thus, task scheduling and resource allocation are mandatory parts of cloud computing research. The efficiency of resource uses depends on the scheduling and load balancing methodologies, rather than the random allocation of resources. Cloud computing is widely used for solving complex tasks (user requests). In solving complex task issues, the use of scheduling algorithm is recommended. Such scheduling algorithms leverage the resources. The proposed system employs features of the Cybershake scientific workflow and the Epigenomics scientific workflow, which are described in Section Input Data.

The major contributions of this paper are summarized as follows.

1. The analytic hierarchy process is modified to rank scientific tasks.

2. To manage the resources given bandwidth constraints and the load on the virtual machine, the proposed system incorporates a version of the existing BATS algorithm that has been modified by introducing BAR system optimization.

3. Bipartite graphs are utilized to map tasks to appropriate virtual machines once the condition is satisfied.

4. A preemption methodology gives us the status of the virtual machine, and a modified divide-and-conquer methodology has been proposed to aggregate the results after tasks preemption.

5. The proposed solution is experimentally investigated using the CloudSim simulator.

The remainder of the paper is organized as follows. Section "Introduction" provides an introduction to cloud computing and its outstanding issues, especially task scheduling and resource allocation. Section "Related work" focuses on related studies that investigate task scheduling and resource allocation. Section "Input data" describes the input data provided to the Cybershake scientific workflows and the Epigenomics scientific workflow. Section "Proposed system" addresses the architecture of the proposed system. Section "Proposed methodology" explains the proposed methodology. Section "Evaluation of the proposed heuristic approach" focuses on evaluating the proposed heuristic approach. Section "Results and discussion" describes the results and discusses the proposed system in comparison with the existing BATS and IDEA algorithms. Finally, concluding remarks and future directions are presented in Section "Conclusion".

\section{Related work}

This section provides a brief review of task scheduling and resource allocation strategies. Many researchers have proposed solutions to overcome the problem of scheduling and resource allocation. However, further improvements can still be made. Tsai et al. [4] proposed a multi-object approach that employs the improved differential evolution algorithm. This existing method provides a cost and time model for cloud computing. However, variations in the tasks are not considered in this approach. Magukuri et al. [5] proposed a load balancing and scheduling algorithm that does not consider job sizes. The authors considered the refresh times of the server in fulfilling requests. Cheng et al. [6] introduced the scheduling of tasks based on a vacation queuing model. This methodology does not show the proper utilization of resources. Lin et al. [7] proposed the scheduling of tasks while considering bandwidth as a resource. A nonlinear programming model has been formed to allocate resources to tasks. Ergu et al. [8] proposed AHP ranking-based task scheduling. Zhu et al. [9] introduced rolling-horizon scheduling architecture to schedule real-time tasks. Authors have illustrated the relationship between task scheduling and energy conservation by resource allocation. Lin et al. [10] proposed scheduling for parallel workloads. Authors have used the FCFS approach to order jobs when resources are available. The proposed system does not focus on aborting the jobs and starvation. Ghanbari et al. [11] proposed a priority-based job scheduling algorithm for use in cloud 
computing. Multi criteria decisions and multiple attributes are considered. Polverini et al. [12] introduced the optimized cost of energy and queuing delay constraints. Alejandra et al. [13] proposed the use of meta-heuristic optimization and particle swarm optimization to reduce execution costs through scheduling. Keshk et al. [14] proposed the use of modified ant colony optimization in load balancing. This method improves the makespan of a job. This system does not consider the availability of resources or the weight of tasks. Shamsollah et al. [15] proposed a system based on a multi-criteria algorithm for scheduling server load. Shamsollah et al. [16] proposed a system based on priority for performing divisible load scheduling that employs analytical hierarchy process. Gougarzi et al. [17] proposed a resource allocation problem that aims to minimize the total energy cost of cloud computing systems while meeting the specified client-level SLAs in a probabilistic sense. Here, authors have applied a reverse approach that applies a penalty if the client does not meet the SLA agreements. Some authors have implemented a heuristic algorithm to solve task scheduling and resource allocation problem described above. Radojevic et al. [18] introduced central load balancing decision model for use in cloud environments; this model automates the scheduling process and reduces the role of human administrators. However, this model is deficient in determining the capabilities of nodes and, configuration details, and the complete system has no backup, thus resulting in a single point of failure. In addition, Ghanbari et al. [19] and Goswami et al. [20] focus on scheduling tasks while considering various constraints. This state-of the art motivates the authors of this study to conduct additional research on task scheduling and resource allocation.

\section{Input data}

\section{Cybershake scientific workflow}

Cloud computing is the service provider paradigm in which users submit requests for execution. Thus, the responsibility of the cloud service provider is to schedule various requests and manage resources efficiently. To the best of the authors' knowledge, most existing work involves scheduling tasks once they enter a task queue. However, the actual procedure of scheduling tasks and resource management begins with how the service provider addresses incoming tasks. The proposed system uses Cybershake scientific workflow data as input tasks [21]. Fig. 1 shows a visualization of the Cybershake scientific workflow, which is used by the Southern California Earthquake Center (SCEC) to characterize earthquake hazards using the Probabilistic Seismic Hazard Analysis (PSHA) technique. It also generates Green strain tensors (GSTs). Table 1 shows the Cybershake seismogram synthesis tasks with their sizes and execution times. The Cybershake is a collection of various node data that are

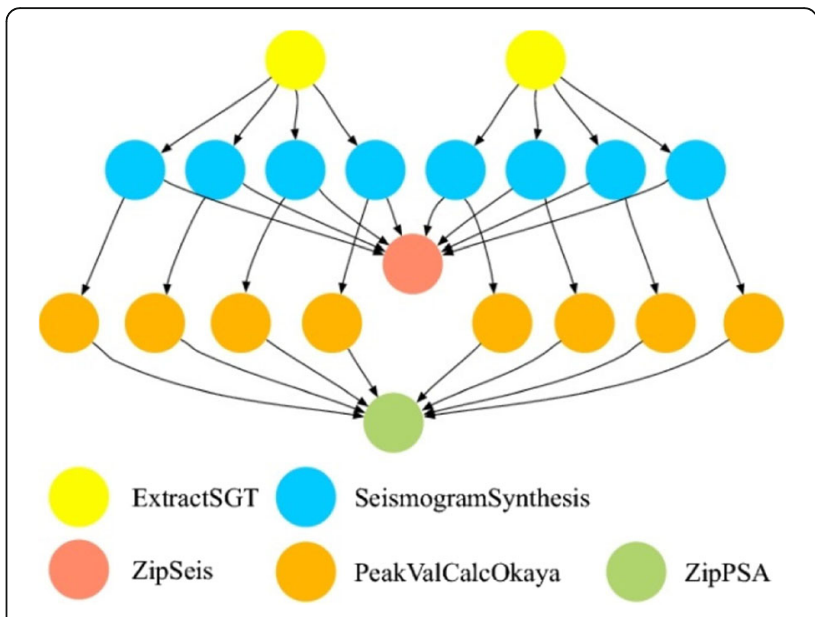

Fig. 1 Cybershake scientific workflow

available for study [22]. The Cybershake scientific workflow sample tasks are available with task size 30,50,100 and 1000. From a computational point of view, the seismogram synthesis tasks are quite demanding. The Cybershake spends a lot of time on seismogram synthesis during its execution. These types of tasks also require large amount of computational resources, such as CPU time, and memory.

Cybershake scientific workflow has been divided into 5 steps.

1. Extract GST - This step of the workflow extracts the GST (Green strain tensor) data for processing.

2. Seismogram synthesis - These tasks are the most computationally intensive. Most of the time spent in running the Cybershake algorithm is employed on this step.

3. ZipSeis - This step aggregates the processed data.

Table 1 Cybershake seismogram synthesis tasks

\begin{tabular}{lll}
\hline Tasks & Size of tasks & Time \\
\hline Task 3 & $62,69,51,663$ & 39.06 \\
Task 5 & $69,47,76,323$ & 38.49 \\
Task 7 & $58,57,63,637$ & 36.27 \\
Task 9 & $53,68,97,326$ & 32.29 \\
Task 11 & $67,05,35,542$ & 62.25 \\
Task 14 & $40,67,28,38,798$ & 96.91 \\
Task 16 & $45,23,96,996$ & 45.60 \\
Task 18 & $50,27,64,231$ & 28.67 \\
Task 20 & $62,41,88,532$ & 24.56 \\
Task 22 & $42,65,77,006$ & 31.05 \\
Task 24 & $51,58,32,878$ & 54.87 \\
Task 26 & $68,14,99,417$ & 23.99 \\
Task 28 & $44,14,51,516$ & 26.46 \\
\hline
\end{tabular}




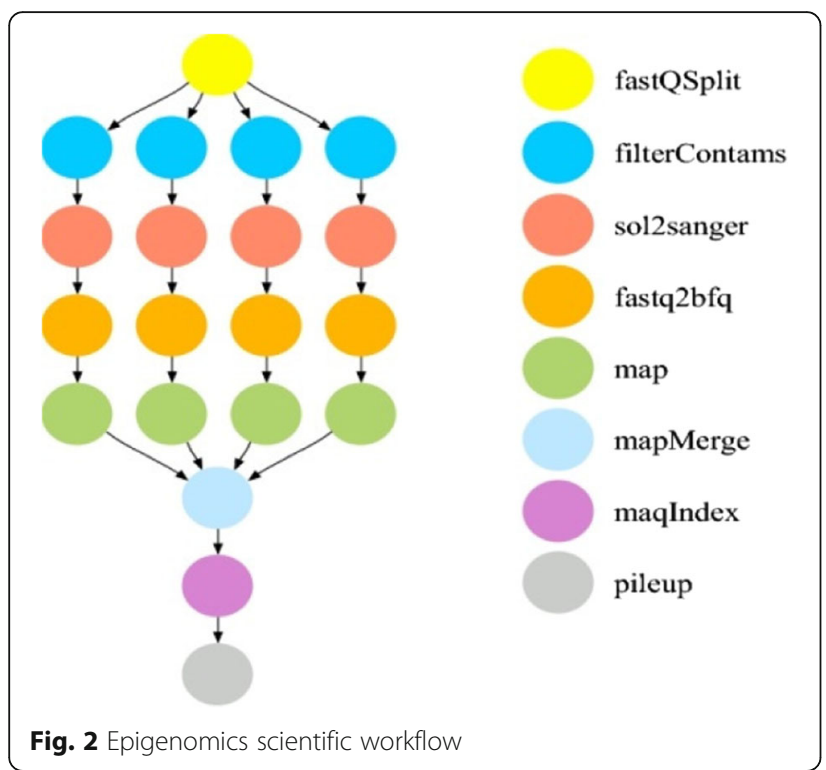

4. PeakValCalcOkaya - The highest-strength values of each seismogram are calculated in this step.

5. ZipPSA - This step aggregates the processed data.

\section{Epigenomics scientific workflow}

Figure 2 shows the Epigenomics scientific workflow [22], which is used to automate the process of genome sequencing. This operation is associated with resourceintensive tasks. The generated data are converted into files and forwarded to Mag system. This process also involves many operations, and these operations are time consuming.

\section{Proposed system}

Figure 3 shows the architecture of the proposed system. In practice, various types and sizes of tasks arrive at the cloud data centers for execution. The proposed system takes the real tasks as an input, as described in Section
3. In general, scientific tasks represent collections of different types and sizes. To manage the tasks that come into a cloud data center, the proposed system uses the analytic hierarchy process (AHP). The primary aim of this proposed system is to manage incoming tasks. Therefore, the proposed system uses the AHP methodology to assign a rank to each task based on its length and run time. The procedure for ranking the tasks for scientific workflows is described in section 5.1. As soon as the tasks are assigned individual rankings, they are collected and arranged into task queues. The tasks in the task queue are strictly arranged following the AHP ranking. Thus, the first stage of the proposed system is completed. Next, in the second stage, the proposed system also addresses the computing resources of cloud data centers, such as CPU, memory and bandwidth using the proposed BATS+BAR optimized allocation methodology. This methodology works as follows. It takes the task to be executed from the task' queue. The assignment of resources and tasks follows the allocation Eq. 4. A detailed explanation is given in section 5.2. This stage is the second part of the procedure in which the allocations of resources have been carried out using BATS+BAR. In the next part, the proposed system uses a preemption methodology, i.e., the preemption method. LEPT continuously checks the load of the virtual machine. If it is exceeded the proposed system then uses a virtual machine status table to determine the current status of other virtual machines (VMs). In this regard, if the current virtual machine is overloaded and others are idle, then such VMs are located. After this identification, the proposed system uses a divideand-conquer methodology, which breaks up the task and distributes it to other virtual machines, as described in detail in section 5.3. In this way, the proposed system has overcome the limitations of BATS in terms of the allocation of resources on the basis of $\mathrm{CPU}$, memory and bandwidth. If any one resource (CPU,

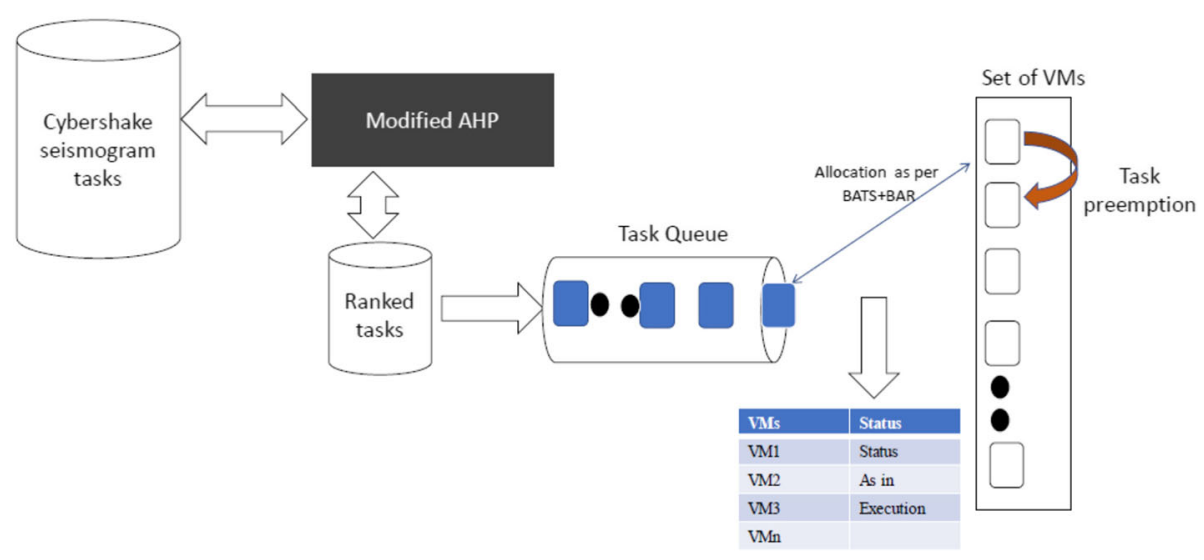

Fig. 3 Proposed system architecture 


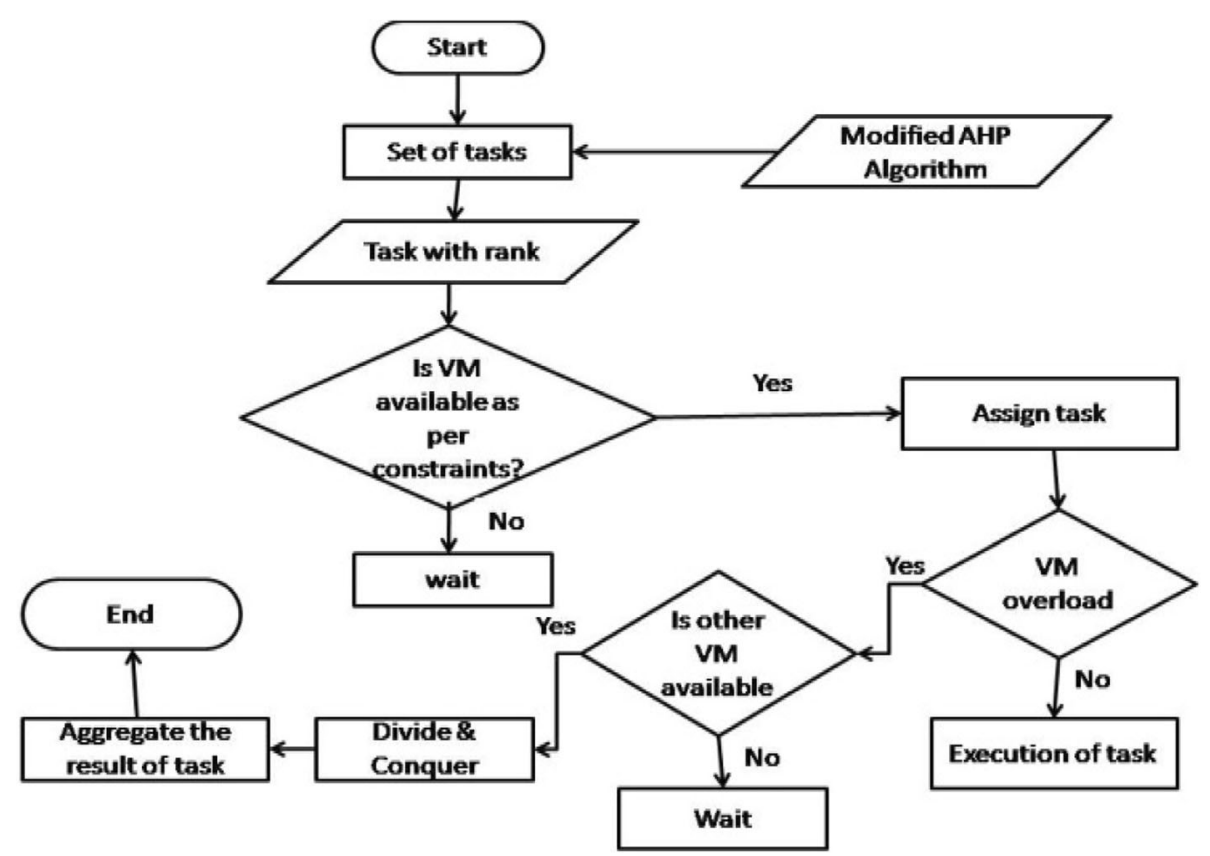

Fig. 4 Proposed system flowchart

memory, bandwidth) is not available in sufficient amounts, then the tasks must wait. In addition, existing systems do not consider preemption, and the inputs to existing systems are tasks of the same size. Fig. 4 presents a flow chart that represents the proposed heuristic approach.

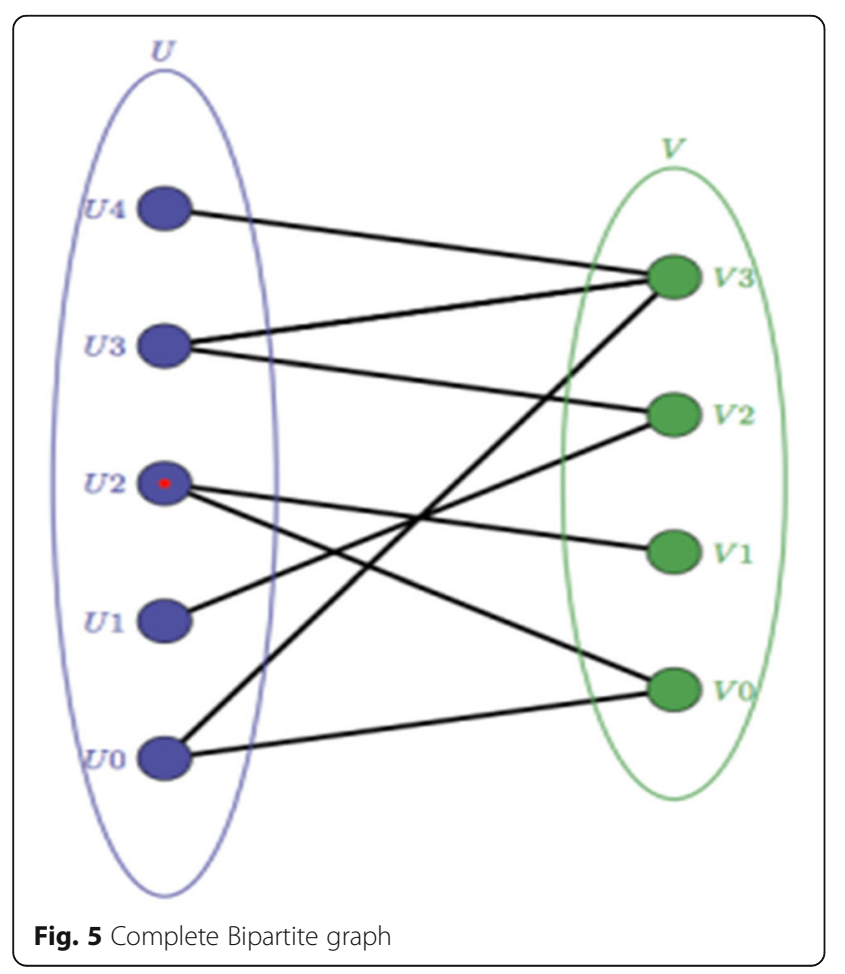

\section{Proposed methodology}

Here, we provide a detailed explanation of the proposed system to overcome the scheduling challenge.

\section{Analytic hierarchy process}

The analytic hierarchy process [23] is designed to solve complex problems with multiple criteria. The proposed system uses this procedure in cloud computing environments to rank the incoming tasks in a certain manner. The proposed system uses scientific workflow tasks, such as those of Cybershake and Epigenomics, for experiments because such require long execution times. Initially, the workflow is divided into five stages, which are introduced in the input data section. Before proceeding with the proposed system, the AHP methodology is applied for the

Table 2 Numerical saaty preferences

\begin{tabular}{ll}
\hline Numerical rating & Judgment preference \\
\hline 9 & Extremely preferred \\
8 & Very strongly to extremely preferred \\
7 & Very strongly preferred to preferred \\
6 & Strongly to very strongly \\
5 & Strongly preferred \\
4 & Moderately to strongly preferred \\
3 & Moderately preferred \\
2 & Equally to moderately preferred \\
1 & Equally preferred \\
\hline
\end{tabular}


Table 3 Summation of each column-I

\begin{tabular}{lllllllll}
\hline Task & Task 3 & Task 5 & Task 7 & Task 9 & Task 11 & Task 14 & Task 16 & Task 18 \\
\hline Task 3 & 1 & 2 & 3 & 4 & $1 / 7$ & $1 / 6$ & $1 / 9$ & 6 \\
Task 5 & $1 / 2$ & 1 & 3 & 4 & $1 / 5$ & $1 / 6$ & $1 / 3$ & 6 \\
Task 7 & $1 / 3$ & $1 / 2$ & 1 & 4 & $1 / 6$ & $1 / 7$ & $1 / 4$ & 6 \\
Task 9 & $1 / 4$ & $1 / 7$ & $1 / 2$ & 1 & $1 / 7$ & $1 / 8$ & $1 / 5$ & 6 \\
Task 11 & 2 & 2 & 2 & 4 & 1 & $1 / 2$ & 3 & 6 \\
Task 14 & 3 & 3 & 3 & 4 & 2 & 1 & 2 & 6 \\
Task 16 & 2 & 2 & 3 & 4 & $1 / 3$ & $1 / 4$ & 1 & 6 \\
Task 18 & $1 / 5$ & $1 / 5$ & $1 / 4$ & $1 / 3$ & $1 / 8$ & $1 / 9$ & $1 / 6$ & 1 \\
Task 20 & $1 / 4$ & $1 / 4$ & $1 / 4$ & $1 / 3$ & $1 / 7$ & $1 / 8$ & $1 / 5$ & $1 / 2$ \\
Task 22 & $1 / 4$ & $1 / 4$ & $1 / 3$ & $1 / 2$ & $1 / 7$ & $1 / 8$ & $1 / 5$ & 6 \\
Task 24 & 3 & 3 & 3 & 4 & $1 / 2$ & $1 / 3$ & 2 & 6 \\
Task 26 & $1 / 5$ & $1 / 5$ & $1 / 5$ & $1 / 4$ & $1 / 8$ & $1 / 9$ & $1 / 6$ & $1 / 3$ \\
Task 28 & $1 / 5$ & $1 / 5$ & $1 / 4$ & $1 / 3$ & $1 / 8$ & $1 / 9$ & $1 / 6$ & $1 / 2$ \\
Sum & $791 / 60$ & $46 / 3$ & $1195 / 60$ & $365 / 12$ & $601 / 112$ & $183 / 56$ & $3229 / 360$ & $338 / 6$ \\
\hline
\end{tabular}

overall Cybershake workflow. The Cybershake workflow is control flow dependent; thus, the second stage will execute only after the execution of the first stage. To evaluate preferences, the proposed system uses the Saaty preference table, which is given in Table 2 with its numerical ratings. To promote understanding while accounting for space limitations, the proposed system divides each calculation table into two parts. The first part extends from Task 3 to Task 18, whereas the other part shows the calculations from Task 20 to Task 28.

Here, the proposed system considers two significant criteria that are involved in scientific tasks; task length and task run time. The comparison numerical ratings are given in Table 2, which is known as the Saaty preference table. Before

Table 4 Summation of each column-II

\begin{tabular}{llllll}
\hline Task & Task 20 & Task 22 & Task 24 & Task 26 & Task 28 \\
\hline Task 3 & 8 & 5 & $1 / 8$ & 9 & 7 \\
Task 5 & 8 & 5 & $1 / 4$ & 9 & 7 \\
Task 7 & 8 & 5 & $1 / 5$ & 9 & 7 \\
Task 9 & 8 & 5 & $1 / 6$ & 9 & 7 \\
Task 11 & 8 & 5 & 3 & 9 & 7 \\
Task 14 & 8 & 5 & 2 & 9 & 7 \\
Task 16 & 8 & 5 & $1 / 2$ & 9 & 7 \\
Task 18 & 8 & $1 / 2$ & $1 / 7$ & 9 & 7 \\
Task 20 & 1 & $1 / 3$ & $1 / 6$ & 9 & $1 / 2$ \\
Task 22 & 8 & 1 & $1 / 6$ & 9 & 7 \\
Task 24 & 8 & 5 & 1 & 9 & 7 \\
Task 26 & $1 / 2$ & $1 / 4$ & $1 / 7$ & 1 & $1 / 3$ \\
Task 28 & 8 & $1 / 3$ & $1 / 7$ & 9 & 1 \\
Sum & $179 / 2$ & $509 / 121$ & $2161 / 280$ & 109 & $431 / 6$ \\
\hline
\end{tabular}

Table 5 Normalization of column value-l

\begin{tabular}{lllllllll}
\hline Tasks & 3 & 5 & 7 & 9 & 11 & 14 & 16 & 18 \\
\hline 3 & $60 / 791$ & $6 / 46$ & $35 / 239$ & $48 / 365$ & $16 / 601$ & $28 / 549$ & $40 / 3229$ & $9 / 84$ \\
5 & $30 / 791$ & $3 / 46$ & $35 / 239$ & $48 / 365$ & $112 / 300$ & $28 / 549$ & $120 / 3229$ & $9 / 84$ \\
7 & $20 / 791$ & $3 / 92$ & $60 / 119$ & $48 / 365$ & $56 / 1803$ & $8 / 183$ & $90 / 3229$ & $9 / 84$ \\
9 & $15 / 791$ & $1 / 46$ & $6 / 239$ & $12 / 365$ & $16 / 601$ & $7 / 183$ & $70 / 3229$ & $9 / 84$ \\
11 & $120 / 791$ & $6 / 46$ & $24 / 239$ & $48 / 365$ & $112 / 601$ & $28 / 183$ & $1080 / 3229$ & $9 / 84$ \\
14 & $180 / 791$ & $9 / 46$ & $35 / 239$ & $48 / 365$ & $224 / 601$ & $56 / 183$ & $720 / 3229$ & $9 / 84$ \\
16 & $120 / 791$ & $6 / 46$ & $35 / 239$ & $49 / 365$ & $112 / 1803$ & $14 / 183$ & $360 / 3229$ & $9 / 84$ \\
18 & $12 / 791$ & $3 / 230$ & $3 / 239$ & $4 / 365$ & $14 / 601$ & $56 / 1647$ & $60 / 3229$ & $6 / 338$ \\
20 & $15 / 791$ & $3 / 184$ & $3 / 239$ & $4 / 365$ & $16 / 601$ & $7 / 183$ & $72 / 3229$ & $3 / 338$ \\
22 & $15 / 791$ & $3 / 184$ & $4 / 239$ & $6 / 365$ & $16 / 601$ & $7 / 183$ & $72 / 3229$ & $9 / 84$ \\
24 & $180 / 791$ & $9 / 46$ & $35 / 239$ & $48 / 365$ & $56 / 601$ & $56 / 549$ & $720 / 3229$ & $9 / 84$ \\
26 & $12 / 791$ & $3 / 230$ & $12 / 119$ & $3 / 365$ & $14 / 601$ & $56 / 1647$ & $60 / 3229$ & $1 / 168$ \\
28 & $12 / 791$ & $3 / 230$ & $3 / 239$ & $4 / 365$ & $14 / 601$ & $56 / 1647$ & $60 / 3229$ & $3 / 338$ \\
Sum & 1 & 0.96 & 0.9723 & 1 & 1.0576 & 0.9817 & 1.09 & 1.005 \\
\hline
\end{tabular}

the actual calculation is begun, the proposed system assigns preference values to the tasks. Here, the preferences associated with the tasks are based on their lengths and the execution times of the different tasks. The proposed system slightly modifies the Saaty table preferences because, as tasks with different ranks are on a server, the ranks of subsequent tasks change, and new rankings must be calculated. The proposed system calculates such rankings of tasks. Tables 3 and 4 show the assignment of Saaty preferences according to comparing the sizes and runtimes of tasks. In the bottom row, the sum of each column is noted.

Tables 5 and 6 show the multiplication of the Saaty preference values by the results arranged in the bottom rows of Tables 3 and 4 and then present the results of adding each column at the bottom.

Table 6 Normalization of column value-II

\begin{tabular}{llllll}
\hline Tasks & 20 & 22 & 24 & 26 & 28 \\
\hline 3 & $16 / 179$ & $60 / 509$ & $35 / 2161$ & $9 / 109$ & $42 / 431$ \\
5 & $16 / 179$ & $60 / 509$ & $70 / 2161$ & $9 / 109$ & $42 / 431$ \\
7 & $16 / 179$ & $60 / 509$ & $65 / 2161$ & $9 / 109$ & $42 / 431$ \\
9 & $16 / 179$ & $60 / 509$ & $140 / 6483$ & $9 / 109$ & $42 / 431$ \\
11 & $16 / 179$ & $60 / 509$ & $840 / 2161$ & $9 / 109$ & $42 / 431$ \\
14 & $16 / 179$ & $60 / 509$ & $560 / 2161$ & $9 / 109$ & $42 / 431$ \\
16 & $16 / 179$ & $60 / 509$ & $140 / 2161$ & $9 / 109$ & $42 / 431$ \\
18 & $16 / 179$ & $16 / 509$ & $40 / 2161$ & $9 / 109$ & $42 / 431$ \\
20 & $2 / 179$ & $4 / 509$ & $140 / 6483$ & $9 / 109$ & $3 / 43$ \\
22 & $16 / 179$ & $12 / 509$ & $140 / 6483$ & $9 / 109$ & $42 / 431$ \\
24 & $16 / 179$ & $60 / 509$ & $280 / 2161$ & $9 / 109$ & $42 / 431$ \\
26 & $1 / 179$ & $3 / 509$ & $40 / 2161$ & $1 / 109$ & $2 / 43$ \\
28 & $16 / 179$ & $4 / 509$ & $40 / 2161$ & $9 / 109$ & $6 / 43$ \\
Sum & 1 & 1.066 & 1.034 & 1 & 1.351 \\
\hline
\end{tabular}


Tables 7 and 8 show the normalized values of Tables 5 and 6, which appear earlier in the manuscript. These tables include average at the bottom. The results show that the summation of each column is equal to 1 .

After this calculation, the rankings of Cybershake seismogram synthesis scientific workflow tasks are given in Table 9.

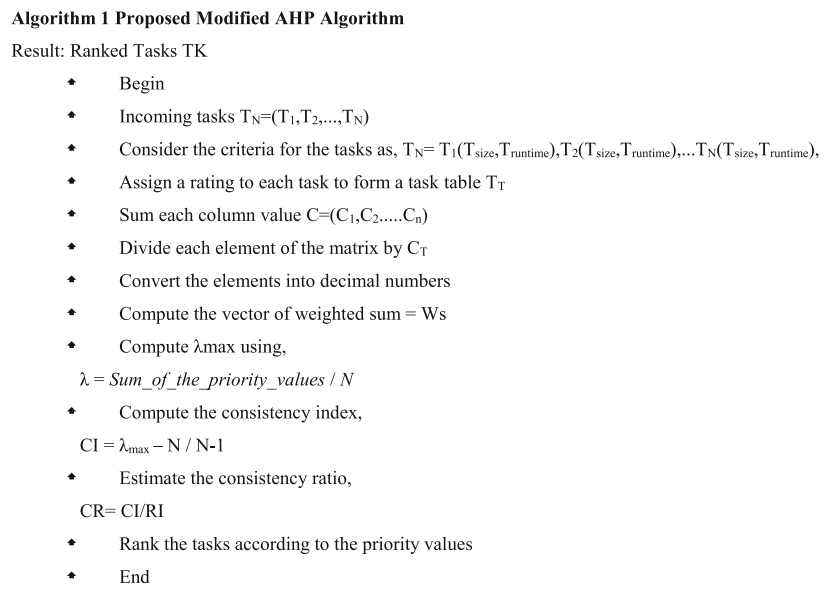

\section{BATS+ BAR system}

The proposed system has two aspects, which involve scheduling tasks and managing resources. Here, we improve upon the BATS algorithm, which was originally proposed by Weiwei Lin [7]. Independent tasks of equal size are considered in the design of this system. However, in allocating resources, the system does not consider the load on virtual machines because the waiting period for the tasks is long. In other cases, one virtual machine is busy while it executes a task, whereas others

Table 7 Compute weighted sum-I

\begin{tabular}{lllllllll}
\hline & 3 & 5 & 7 & 9 & 11 & 14 & 16 & 18 \\
\hline Task 3 & 0.075 & 0.086 & 0.146 & 0.131 & 0.026 & 0.051 & 0.012 & 0.107 \\
Task 5 & 0.037 & 0.086 & 0.146 & 0.131 & 0.037 & 0.051 & 0.037 & 0.107 \\
Task 7 & 0.025 & 0.032 & 0.050 & 0.131 & 0.031 & 0.043 & 0.027 & 0.107 \\
Task 9 & 0.018 & 0.021 & 0.025 & 0.032 & 0.026 & 0.038 & 0.021 & 0.107 \\
Task 11 & 0.151 & 0.130 & 0.100 & 0.131 & 0.186 & 0.153 & 0.334 & 0.107 \\
Task 14 & 0.227 & 0.195 & 0.146 & 0.131 & 0.372 & 0.306 & 0.222 & 0.107 \\
Task 16 & 0.151 & 0.021 & 0.146 & 0.131 & 0.062 & 0.076 & 0.111 & 0.107 \\
Task 18 & 0.015 & 0.013 & 0.012 & 0.010 & 0.023 & 0.034 & 0.018 & 0.017 \\
Task 20 & 0.018 & 0.016 & 0.012 & 0.010 & 0.026 & 0.038 & 0.022 & 0.008 \\
Task 22 & 0.018 & 0.016 & 0.016 & 0.016 & 0.026 & 0.038 & 0.022 & 0.107 \\
Task 24 & 0.227 & 0.195 & 0.146 & 0.131 & 0.093 & 0.102 & 0.222 & 0.107 \\
Task 26 & 0.015 & 0.013 & 0.010 & 0.008 & 0.023 & 0.034 & 0.018 & 0.005 \\
Task 28 & 0.015 & 0.013 & 0.012 & 0.010 & 0.023 & 0.034 & 0.018 & 0.008 \\
Sum & 1 & 0.96 & 0.972 & 1 & 1.057 & 0.981 & 1.09 & 1.005 \\
\hline
\end{tabular}

Table 8 Compute weighted sum-II

\begin{tabular}{lllllll}
\hline & 20 & 22 & 24 & 26 & 28 & Average of I and II \\
\hline Task 3 & 0.089 & 0.117 & 0.016 & 0.082 & 0.097 & 0.079 \\
Task 5 & 0.089 & 0.117 & 0.032 & 0.082 & 0.097 & 0.116 \\
Task 7 & 0.089 & 0.117 & 0.026 & 0.082 & 0.097 & 0.065 \\
Task 9 & 0.089 & 0.117 & 0.021 & 0.082 & 0.097 & 0.053 \\
Task 11 & 0.089 & 0.117 & 0.388 & 0.082 & 0.097 & 0.158 \\
Task 14 & 0.089 & 0.117 & 0.259 & 0.082 & 0.097 & 0.180 \\
Task 16 & 0.089 & 0.117 & 0.064 & 0.082 & 0.097 & 0.096 \\
Task 18 & 0.089 & 0.011 & 0.018 & 0.082 & 0.097 & 0.033 \\
Task 20 & 0.011 & 0.007 & 0.021 & 0.082 & 0.069 & 0.026 \\
Task 22 & 0.089 & 0.023 & 0.021 & 0.082 & 0.097 & 0.055 \\
Task 24 & 0.089 & 0.117 & 0.129 & 0.082 & 0.097 & 0.133 \\
Task 26 & 0.005 & 0.005 & 0.018 & 0.009 & 0.046 & 0.016 \\
Task 28 & 0.089 & 0.007 & 0.018 & 0.082 & 0.139 & 0.036 \\
Sum & 1 & 1.066 & 1.034 & 1 & 1.351 & 1.046 \\
\hline
\end{tabular}

are occupied and waiting for jobs. The bar systems (BSs) algorithm was proposed by Acebo and Rosa (2008) [24]. The social behavior of bartenders is the basis of BS systems. Swarm intelligence has added an optimization aspect to BS. In a bar, bartenders must act in a highly dynamic, asynchronous and time-critical environment, and no obvious greedy strategy (such as serving the best customer first, serving the nearest customer first or serving the first-arriving customer first) gives good results. Thus, multi-agent systems provide a good framework within which to address the challenge of developing a new class of adaptive and robust systems. In general, the crucial step in the BS algorithm is the choice of the task that the agent must execute in the next time step. In $\mathrm{BSs}$, agents acting as bartenders, operate concurrently in

Table 9 AHP rankings of cybershake seismogram synthesis tasks

\begin{tabular}{|c|c|c|}
\hline Rank & Before AHP & After AHP \\
\hline 1 & Task 5 & Task 26 \\
\hline 2 & Task 3 & Task 20 \\
\hline 3 & Task 9 & Task 18 \\
\hline 4 & Task 7 & Task 28 \\
\hline 5 & Task 20 & Task 09 \\
\hline 6 & Task 22 & Task 22 \\
\hline 7 & Task 11 & Task 07 \\
\hline 8 & Task 24 & Task 03 \\
\hline 9 & Task 16 & Task 16 \\
\hline 10 & Task 26 & Task 05 \\
\hline 11 & Task 14 & Task 24 \\
\hline 12 & Task 28 & Task 11 \\
\hline 13 & Task 18 & Task 14 \\
\hline
\end{tabular}


an environment in a synchronous manner; that is, they execute tasks by deciding which drinks to pour. After an initial phase, the "bartenders" make their decisions according to different problem-dependent properties (e.g. weight, speed, location, response time, maximum load, etc.), instead of making decisions randomly. Over time, if an agent is unable to adapt the environment to the preconditions of the task (such as the cost for the agent to execute the task in the current state of the environment) or if it is unable to carry the task out by itself, it will be eliminated. To overcome this behavior, we propose modifying BATS by adding a BAR system. The procedure is as follows:

1. Aggregate all of the task information that is ordered by rank.

2. Virtual machine (server) information is collected. This information includes the initial load on the virtual machine, its bandwidth and the time required to process the tasks on the server.

3. A bipartite graph is generated with the number of tasks. The ranking priorities, can be used to constructed a graph, by which each task is allocated to a virtual machine.

The Load on the virtual machine(S) is calculated as,

$$
\boldsymbol{L}^{i n i}=\boldsymbol{L}_{s}^{i n i} \mid s \subset \boldsymbol{S}
$$

The bandwidth is calculated as,

$$
D B_{w}=b^{\prime} \boldsymbol{i}<=b \boldsymbol{i}
$$

The total time taken to process the tasks is calculated as,

$$
\boldsymbol{L}_{s}^{f i n}(\boldsymbol{\alpha})=\boldsymbol{L}_{s}(\boldsymbol{\alpha})
$$

Where, $(\alpha)=$ any task.

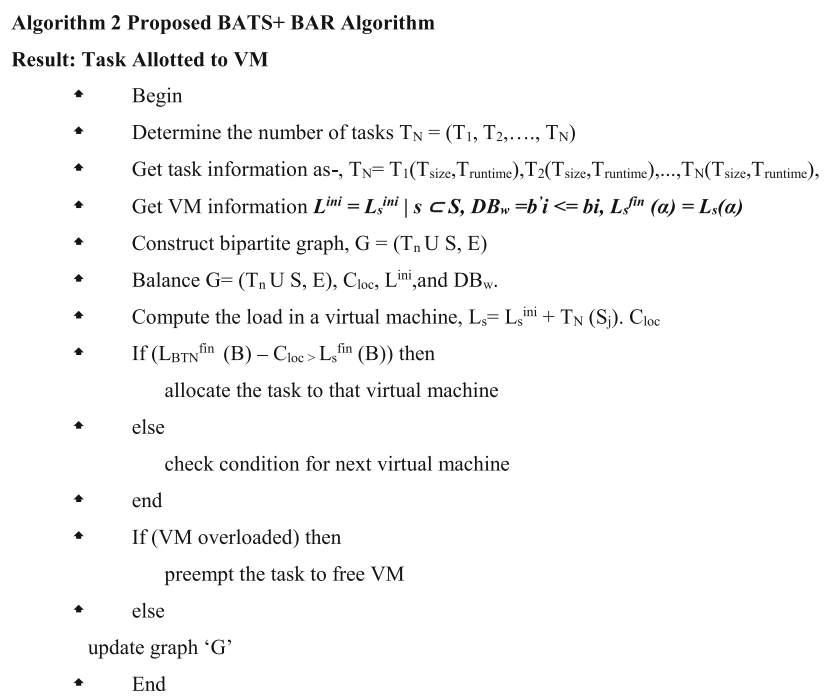

\section{Bipartite graph}

A bipartite graph is produced based on the following conditions:

1. A bipartite graph is constructed as-, $G=\left(T_{n} U S, E\right)$ in which ' $\mathrm{T}_{\mathrm{n}}$ ' represents the number of tasks, ' $\mathrm{S}$ ' represents the servers, and ' $E \subseteq \mathrm{T} X \mathrm{~S}$ ' that is, the set of edges that are present between the task and the server. An edge represents the tasks ' $\mathrm{Ti} \subset \mathrm{Tn}$ ', which are present on virtual machine 's $\subseteq S$ '. A graph is constructed using bipartite graph with the number of tasks.

2. Balance the constructed graph with constraints including the local cost, the initial load and the bandwidth.

3. Based on the local cost and the initial load we compute the total load on the virtual machine.

$$
\boldsymbol{L}_{s=} \boldsymbol{L}_{s}^{i n i}+\boldsymbol{T}_{N}\left(\boldsymbol{S}_{j}\right) \cdot \boldsymbol{C}_{l o c}
$$

4. Next, we apply the condition represented by Eq. 4. If this condition is satisfied, then we allocate the tasks to that particular virtual machine. If this condition is not satisfied by that virtual machine, then we move on the next server and check this condition.

5. After allocating the tasks, the constructed bipartite is updated if any task remain to be processed. Fig. 5 is the bipartite graph of the set of virtal machine and set of resources.

\section{After allocating the tasks, the constructed bipartite is updated if any task remain to be processed Preemption of the task}

As described in earlier sections, the proposed system ranks the tasks and allocates them as per the constraints of bandwidth and load on the virtual machine. The proposed system next checks the tasks' preemption conditions according to the LEPT policy. Before a task is preempted, we must check the status of the existing virtual machine (i.e. whether it is free or busy).

The system considers situations in which the resourceintensive tasks are still running on allocated virtual machines while other VMs are waiting for tasks at the same time. Therefore, the tasks must be distributed

Table 10 Data center information

\begin{tabular}{lll}
\hline Sr. No. & Characteristic & Value \\
\hline 1 & Number of data center & 1 \\
2 & Number of hosts & 1 \\
3 & Number of processing units & 4 \\
4 & Processing capacity & 9600 mips \\
5 & Storage capacity & 11 TB \\
6 & Total amount of RAM & $40 \mathrm{~GB}$ \\
\hline
\end{tabular}


Table 11 Data center configuration details

\begin{tabular}{lll}
\hline Sr. No. & Characteristic & Value \\
\hline 1 & Allocation policy & BATS +BAR \\
2 & Architecture & X86 \\
3 & Operating system & Linux \\
4 & Hypervisor & Xen \\
5 & Upper threshold & 0.8 \\
6 & Lower threshold & $0.2 \mathrm{~GB}$ \\
7 & VM migration & Enabled \\
8 & Monitoring interval & 180 \\
\hline
\end{tabular}

among the free VMs. However, we must first consider how and when to preempt tasks.

Here, we propose a mathematical description of the preemption scenario,

- Given the ranking of the tasks, they are allocated to the VMs using a bipartite graph, but the issue is now one oftime,

- Suppose 'P1'-'VM1' has complete 't1', or VMn-1 has completed at time $\sum \mathrm{Pn}-1$. However, one VM is stillrunning with high-priority tasks, and its processing time is also greater.

- Therefore, we preempt the task per the following methodology. Before preempting, we should check the status of the VM (i.e. whether it is free or busy).

$$
\begin{aligned}
\mathrm{V}=\min _{\mathrm{tn}, \mathrm{tm}}\left(\frac{1}{\lambda n+\lambda m}\right. & +\frac{\lambda n}{\lambda n+\lambda m}+V \\
& \left.*(\{n\})+\frac{\lambda n}{\lambda n+\lambda m} \mathrm{~V} *(\{\mathrm{~m}\})\right)
\end{aligned}
$$

Here

- $\mathrm{V}(\mathrm{t})$ denotes the expected value of the minimum remaining time needed to finish all of the jobs given the set

$$
\mathrm{t}=\left\{\mathrm{t} 1,---, \mathrm{t}_{\mathrm{n}}\right\}
$$

Table 12 Host configuration details

\begin{tabular}{lll}
\hline Sr. No. & Characteristic & Value \\
\hline 1 & RAM & $40,000 \mathrm{MB}$ \\
2 & Bandwidth & $10,00,000$ \\
3 & Operating System & Linux \\
4 & Hypervisor & Xen \\
5 & Upper threshold & 0.8 \\
6 & Lower Threshold & $0.2 \mathrm{~GB}$ \\
7 & VM Migration & Enabled \\
8 & Monitoring Interval & 180 \\
\hline
\end{tabular}

Table 13 Customer configuration details

\begin{tabular}{lll}
\hline Sr. No. & Information & Contains \\
\hline 1 & User & 1 \\
2 & Cloudlets sent per minutes & 50 \\
3 & Avg. length of cloudlet & 50,000 \\
4 & Avg. cloudlet file size & 500 bytes \\
5 & Avg. cloudlet output size & 500 bytes \\
\hline
\end{tabular}

- $\mathrm{V}^{*}(\mathrm{t})$ denotes the time quantity $\mathrm{t}=\{\mathrm{t} 1$,

- $\mathrm{V}^{*}$ denotes the expected value of the remaining completion time when no task has yet been completed i.e., all tasks are in the running state.

- $\lambda_{\text {tn }}$ denotes the experimentally distributed time required for task $t_{n}$

- $\lambda_{\mathrm{tm}}$ denotes the experimentally distributed time required for task $t_{m}$.

$$
\left[\frac{1}{\lambda_{t n}+\lambda_{t m}}+\frac{\lambda t n}{\lambda_{t n}+\lambda_{t m}} V *(\{t n\})\right]
$$

Eq. 6 is the probability that task $\mathrm{n}(\mathrm{m})$ is the first task to be completed, multiplied by the expected remaining time needed to complete the $t_{n-1}$ tasks.

$$
\frac{\lambda_{t m}}{\lambda_{t n}+\lambda_{t m}} V^{*}(\{\mathrm{~m}\})
$$

We can rewrite Eq. 5 in the following way:,

$$
\begin{aligned}
0=\min _{n, m}\left(1+\lambda_{t n}\left(V^{*}(\{t n\})-V^{*}\right)+\lambda_{t m}\left(V^{*}(\{t n\})-V^{*}\right)\right. \\
\left.+\left(\lambda_{t n}+\lambda_{t m}\right)\left(V^{*}-V\right)\right)
\end{aligned}
$$

Because $\lambda 1$ and $\lambda 2$ are the two smallest $\lambda t$ values, $\mathrm{V}^{*} \geq \mathrm{V}$. The last term is minimized by $\mathrm{tn}, \mathrm{tm}=1,2$ of the second and third terms.

Table 14 Customer configuration details

\begin{tabular}{lll}
\hline Sr. No. & Information & Contains \\
\hline 1 & Number of VMs & 20 \\
2 & Avg. image size & 1000 bytes \\
3 & Avg. RAM & $512 \mathrm{MB}$ \\
4 & Avg. bandwidth & $1,000,000 \mathrm{Mbps}$ \\
5 & Procedure element & 1 \\
6 & Priority & 1 \\
7 & Hypervisor & Xen \\
8 & Scheduling policy & Dynamic workload \\
\hline
\end{tabular}




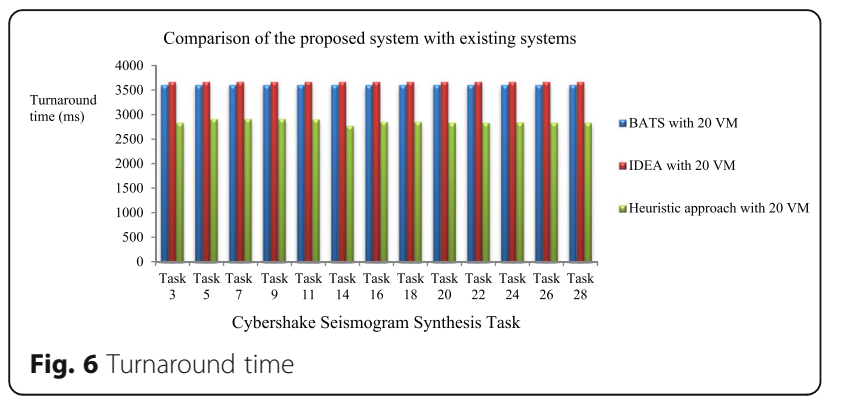

$$
\mathrm{Ctn}=\lambda_{\ln }\left(V^{*}(\{t n\})-V^{*}\right)
$$

Moreover, let,

$$
D_{t_{n}, m}=C_{t_{n}}-C_{t_{m}}
$$

Here

- $C_{\mathrm{tn}}$ denotes the time at which the first VM has finish its task execution

- $\mathrm{C}_{\mathrm{tm}}$ denotes the last task completed by another VM

- D denotes the difference between the completion of the first task and that of the last task i.e., in Eq. 10

Substituting for $\mathrm{C}_{\mathrm{tn}}$ and $\mathrm{C}_{\mathrm{tm}}$. Eq. 10 becomes,

$$
\left.\lambda_{t_{n}}\left(V^{*}(\{\mathrm{n}\})-V^{*}\right)+\lambda_{t m}\left(\mathrm{~V}^{*}\{\mathrm{~m}\}\right)-\mathrm{V}^{*}\right)
$$

Equation 11 is minimized by $\mathrm{j}, \mathrm{k}=1,2$ Let,

$$
\lambda_{t_{n}} \angle \lambda_{t_{m}} \cong \mathrm{C}_{\mathrm{tn}} \leq \mathrm{C}_{\mathrm{tm}}
$$

Therefore, we must improve $\mathrm{D}$ to obtain better results. $C_{t n}$ and $D_{t n}, m$ are considered to be functions of $\lambda 1----\lambda \mathrm{n}$.

Assuming tasks $t_{n}$ and $t_{m}$ are not members of set $t$, we define $C_{t n}(t)$ and $D_{t n, m}(t) ; C_{t n}$ and $D_{t n, m}$

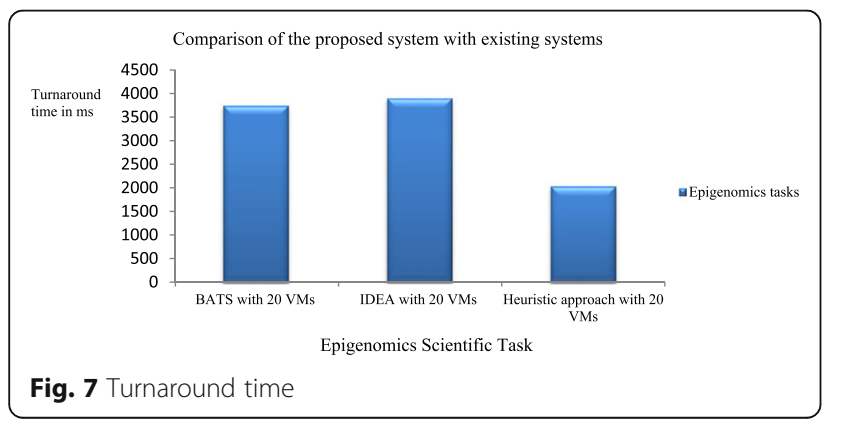

Table 15 Comparison of the proposed heuristic approach with the BATS and IDEA frameworks in terms of TAT in ms

\begin{tabular}{llll}
\hline Tasks & $\begin{array}{l}\text { BATS with } \\
\text { 20 VM }\end{array}$ & $\begin{array}{l}\text { IDEA with } \\
\text { 20 VM }\end{array}$ & $\begin{array}{l}\text { Heuristic approach } \\
\text { with 20 VM }\end{array}$ \\
\hline Task 3 & 3599.29 & 3666.27 & 2832.94 \\
Task 5 & 3599.29 & 3666.27 & 2914.42 \\
Task 7 & 3599.29 & 3666.27 & 2913.87 \\
Task 9 & 3599.29 & 3666.27 & 2911.75 \\
Task 11 & 3599.29 & 3666.27 & 2907.67 \\
Task 14 & 3599.29 & 3666.27 & 2772.11 \\
Task 16 & 3599.29 & 3666.27 & 2857.89 \\
Task 18 & 3599.29 & 3666.27 & 2855.97 \\
Task 20 & 3599.29 & 3666.27 & 2833.36 \\
Task 22 & 3599.29 & 3666.27 & 2834.72 \\
Task 24 & 3599.29 & 3666.27 & 2841.49 \\
Task 26 & 3599.29 & 3666.27 & 2832.86 \\
Task 28 & 3599.29 & 3666.27 & 2833.96 \\
\hline
\end{tabular}

$$
\mathrm{C}_{\mathrm{tn}}(\mathrm{t})=\lambda_{t_{n}}\left(\mathrm{~V}^{*}\left(\mathrm{t} \quad \mathrm{U} \mathrm{t}_{\mathrm{n}}\right)-\mathrm{V}^{*}(\mathrm{t})\right)
$$

Before, the procedure can continue, a number of identities must be formally described. If $t_{n}$ and $t_{m}$ are the two smallest tasks in set $\mathrm{J}$, the LEPT process tasks $t_{n}$ and $t_{m}$ will be executed first.

The following condition is the first task completion result.

$$
\begin{aligned}
\mathrm{V}^{*}(\mathrm{t})= & \frac{1}{\lambda_{t n}+\lambda_{t m}}+\frac{\lambda_{t n}}{\lambda_{t n}+\lambda_{t m}} \boldsymbol{V}^{*}\left(\mathrm{t} \mathrm{U}\left\{\mathrm{t}_{\mathrm{n}}\right\}\right) \\
& +\frac{\lambda_{t n}}{\lambda_{t n}+\lambda_{t m}} \boldsymbol{V}^{*}\left(\mathrm{t} \mathrm{U}\left\{\mathrm{t}_{\mathrm{n}}\right\}\right)
\end{aligned}
$$

Equation 14 can be rewritten as follows:

$$
\begin{aligned}
\left(\lambda_{t n}+\lambda_{t m}\right) \boldsymbol{V}^{*}(\mathrm{t})= & 1+\lambda_{t_{n}} \boldsymbol{V}^{*}\left(\mathrm{t} \mathrm{U}\left(\mathrm{t}_{\mathrm{n}}\right)\right) \\
& +\lambda_{t m} \boldsymbol{V}^{*}\left(\mathrm{t} \mathrm{U}\left(\mathrm{t}_{\mathrm{m}}\right)\right)
\end{aligned}
$$

Table 16 Comparison of the proposed heuristic approach with the BATS and IDEA frameworks in term of TAT in ms

\begin{tabular}{lll}
\hline BATS with 20 VMs & IDEA with 20 VMs & Heuristic approach with $20 \mathrm{VMs}$ \\
\hline 3738 & 3895 & 2033.72 \\
\hline
\end{tabular}




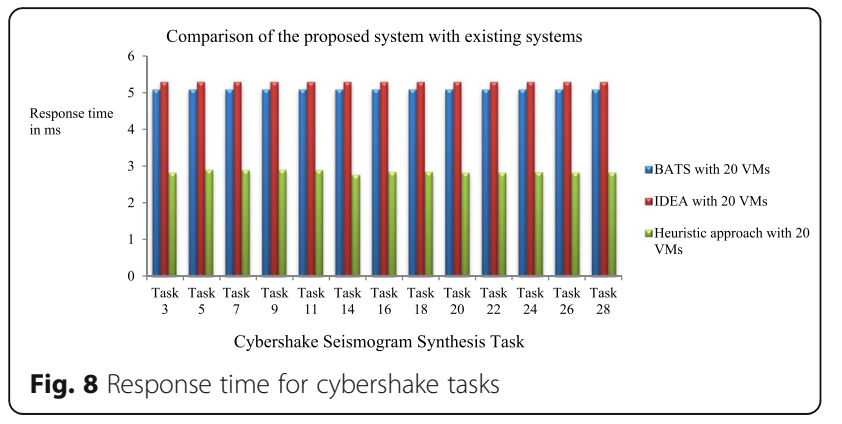

Similarly, the exponentially distributed processing times for 3 tasks are approximately $\lambda 1, \lambda 2, \lambda 3$. Next, we substitute these values into Eq. 13,

Given $t_{n}=\lambda 1, t_{m}=\lambda 2$, and $t_{p}=\lambda 3$, Eq. 13 becomes,

$$
\begin{aligned}
(\lambda 1+\lambda 2+\lambda 3) C_{1}= & \lambda 1(\lambda 1+\lambda 2+\lambda 3) \boldsymbol{V}^{*}(\{1\}) \\
& -\lambda 1(\lambda 1+\lambda 2+\lambda 3) \boldsymbol{V}^{*}
\end{aligned}
$$

$$
\begin{aligned}
(\lambda 1+\lambda 2+\lambda 3) \mathrm{C}_{1}= & \lambda 1\left(1+\lambda 1 V^{*}\{1\}+\lambda 2 V^{*}\{1,2\}\right. \\
& \left.+\lambda 3 V^{*}\{1,3\}\right) \\
& -\lambda 1\left(1+\lambda 1 V^{*}\{1\}\right. \\
& \left.+\lambda 2 V^{*}\{2\}+\lambda 3 V^{*}\{3\}\right)
\end{aligned}
$$

$$
\begin{aligned}
(\lambda 1+\lambda 2+\lambda 3) \mathrm{C}_{1}= & \lambda 1\left(\lambda 3 \boldsymbol{V}^{*}\{1,3\}-\lambda 3 \boldsymbol{V}^{*}\{3\}\right. \\
& +\lambda 2\left(\lambda 1 \boldsymbol{V}^{*}\{1,2\}\right) \\
& -\lambda 1 \boldsymbol{V}^{*}\{2\}+\lambda 3 \boldsymbol{A 1}
\end{aligned}
$$

Thus, Eq. 18 gives the value of the respective $\lambda$.

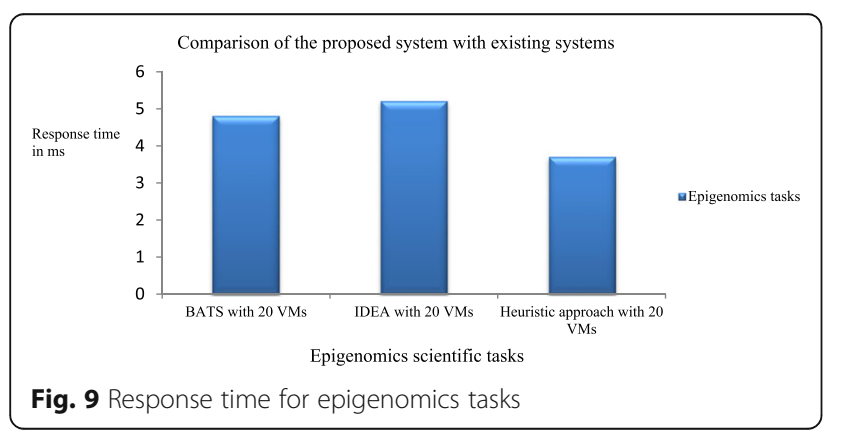

Table 17 Comparison of proposed heuristic approach with the BATS and IDEA frameworks in terms of RT in ms

\begin{tabular}{llll}
\hline Tasks & $\begin{array}{l}\text { BATS with } \\
\text { 20 VMs }\end{array}$ & $\begin{array}{l}\text { IDEA with } \\
\text { 20 VMs }\end{array}$ & $\begin{array}{l}\text { Heuristic approach } \\
\text { with 20 VMs }\end{array}$ \\
\hline Task 3 & 5.1 & 5.3 & 2.83 \\
Task 5 & 5.1 & 5.3 & 2.91 \\
Task 7 & 5.1 & 5.3 & 2.9 \\
Task 9 & 5.1 & 5.3 & 2.91 \\
Task 11 & 5.1 & 5.3 & 2.90 \\
Task 14 & 5.1 & 5.3 & 2.77 \\
Task 16 & 5.1 & 5.3 & 2.85 \\
Task 18 & 5.1 & 5.3 & 2.85 \\
Task 20 & 5.1 & 5.3 & 2.83 \\
Task 22 & 5.1 & 5.3 & 2.83 \\
Task 24 & 5.1 & 5.3 & 2.84 \\
Task 26 & 5.1 & 5.3 & 2.83 \\
Task 28 & 5.1 & 5.3 & 2.83 \\
\hline
\end{tabular}

$$
(\lambda 1+\lambda 2+\lambda 3) C_{1}=\lambda 1\left(\lambda 3 V^{*}\{1,3\}-\lambda 3 V^{*}\{3\}\right.
$$$$
+\lambda 2\left(\lambda 1 V^{*}\{1,2\}\right)-\lambda 1 V^{*}\{2\}+\lambda 3 A 1
$$

Alternatively,

$$
\begin{aligned}
& (\lambda 1+\lambda 2) C_{1}=\lambda_{1} \mathrm{C} 3(1)+\lambda_{2} C_{1}(2) \\
& (\lambda 1+\lambda 2) C_{2}=\lambda_{1} C_{2}(1)+\lambda_{2} C_{3}(2) \\
& (\lambda 1+\lambda 2) C_{t}=\lambda_{1} C_{t}(1)+\lambda_{2} C_{t}(2)
\end{aligned}
$$

For,

$T=1,2--\mathrm{n}$.

Thus,

$\mathrm{D}_{12}=\mathrm{C}_{\mathrm{t} 1}-\mathrm{C}_{\mathrm{t} 2}$ if

$$
D 12=\frac{\lambda_{t_{1}}}{\lambda_{t_{1}}+\lambda_{t_{2}}} D_{32}(1)+\frac{\lambda_{t_{2}}}{\lambda_{t_{1}}+\lambda_{t_{2}}} D_{13}(2)
$$

Table 18 Comparison of the proposed heuristic approach with the BATS and IDEA frameworks in term of RT in ms BATS with $20 \mathrm{VMs}$ IDEA with $20 \mathrm{VMs}$ Heuristic approach with $20 \mathrm{VMs}$ \begin{tabular}{lll}
\hline 4.8 & 5.2 & 3.7
\end{tabular} 


$$
D 2 t=\frac{\lambda_{t_{1}}}{\lambda_{t_{1}}+\lambda_{t_{2}}} D_{2 t}(1)+\frac{\lambda_{t_{2}}}{\lambda_{t_{1}}+\lambda_{t_{2}}} D_{3 t}(2)
$$

We now assume as a hypothesis that

$$
\lambda_{t_{n}}<\lambda_{t_{n}} \text { and } \lambda 1 \ldots \ldots \leq \lambda n
$$

In that case,

$$
\mathrm{D}_{\mathrm{n}, \mathrm{m}} \leq 0
$$

and,

$$
\frac{d D 12}{d \lambda_{t 1}} \geq 0
$$

In the second part of the proof, two in equalities are shown by induction on ' $n$ ', when $n=2$

$$
\mathrm{Dt}_{\mathrm{n}, \mathrm{m}}=\frac{\lambda_{t_{n}}-\lambda_{t_{m}}}{\lambda_{t n}+\lambda_{t m}}
$$

The two inequalities can be easily produced.

Assume that the two inequalities of the induction hypothesis hold when there are fewer than ' $n$ ' tasks remaining to be processed. The induction hypothesis then implies that $D_{t 13}(2)$ and $D_{t 23}(1)$ are non-positive when there are ' $n$ ' tasks remaining to be completed. It also requires

$$
\frac{d D t 13\{2\}}{d \lambda_{t 1}} \geq 0
$$

The inequality presented in Eq. 21 has the following implications.

If increases, then $\mathrm{dD}_{\mathrm{t} 13}(2)$ increases. The moment $\lambda \mathrm{t} 1$ reaches the value of $\lambda \mathrm{t} 2$ tasks 1 and 2 are interchangeable. Here, instead of changing task 2 and task 1 we break the respective tasks and migrate them onto other virtual machine.

\section{Divide-and-conquer methodology}

After, the tasks have been preempted, we apply the divide-and-conquer methodology by following the steps shown below.

\section{Evaluation of the proposed heuristic approach Experimental setup}

The proposed heuristic approach is simulated on a cloud computing environment [25] that provides a real-time

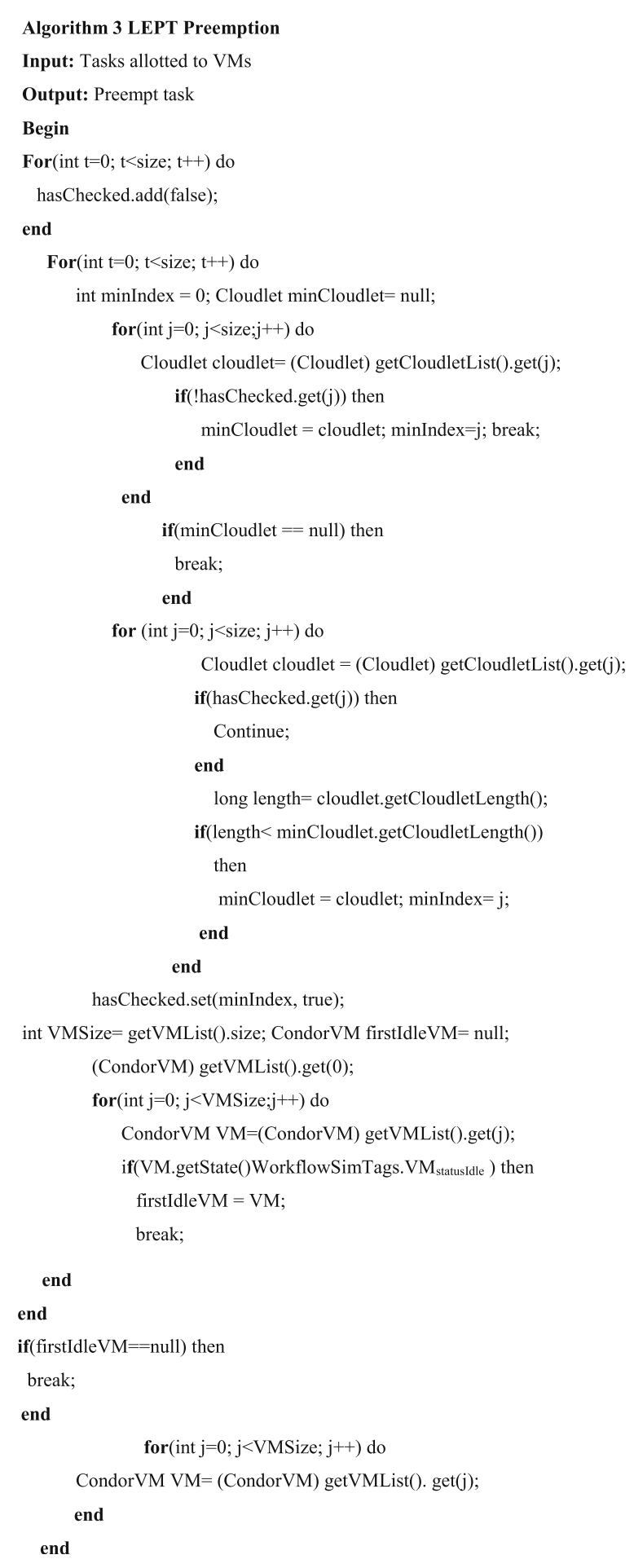

cloud computing scenario. The configuration details of the data center used in the customized simulation setup are given in Table 10 and consist of general information on the data centers, such as the number of data centers, the number of hosts, the number of processing units, 


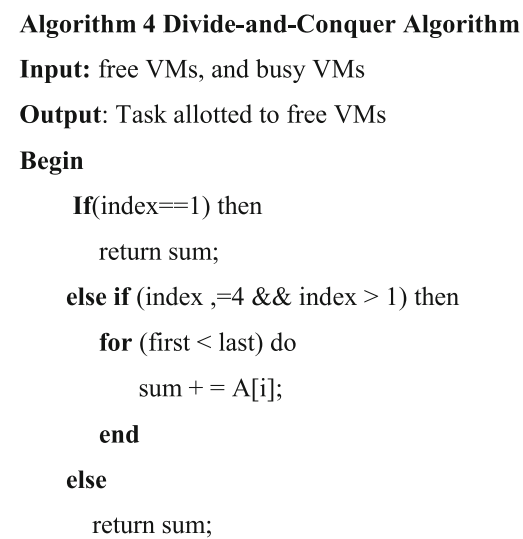

return (sumArray(first, last/2, A) + sumArray (last/2, A.length, A));

and capacity. Every data center component generates a set of strategy for allocating bandwidth, memory and storage devices for hosts and virtual machines. Table 11 shows the configuration for the data center, including its allocation policy, architecture, OS, hypervisor, scheduling and monitoring interval, and threshold value, among other properties. The host in the data center is built with the configuration such as RAM, bandwidth, storage capacity, power, processing elements etc. of the given task, the processing of which by a data center is listed in Table 12. Table 13 provides the details of the customer configuration. Table 14 provides a detailed description of the virtual machines.

\section{Results and discussion}

This section briefly describes the performance of the proposed heuristic approach.

\section{Evaluation of turnaround time}

To check the performance of our proposed heuristic approach, we first apply the algorithm to Cybershake seismogram synthesis and Epigenomics scientific tasks, which are described in the input data section. The performance of the proposed heuristic approach is evaluated, in terms of the turnaround time, which is the span of time from the submission of the task to the

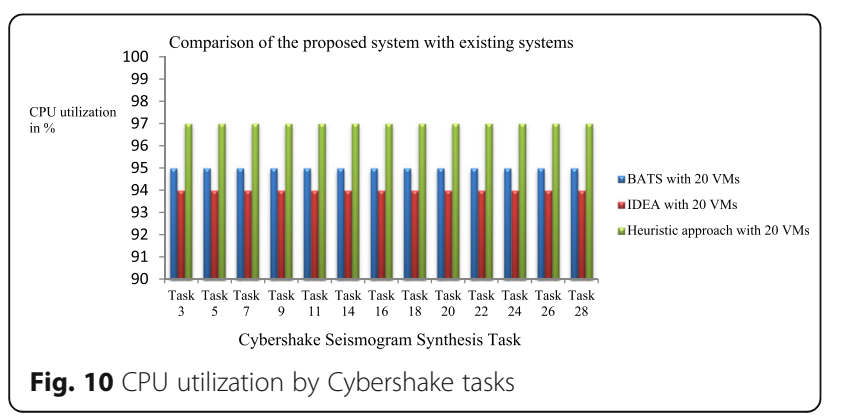

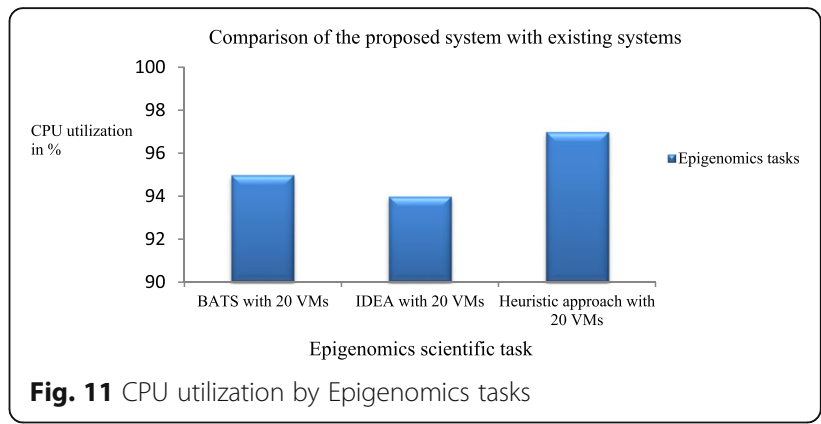

completion of the task. In Fig. 6 the Cybershake tasks are shown on the $\mathrm{X}$ axix, whereas time shown on the $\mathrm{Y}$ axis. When we compare our proposed heuristic approach with the existing BATS [7] and IDEA [4] frameworks, we find that our approach displays reduced turnaround time. Fig. 7 illustrates the results for the Epigonomics tasks, for which the turnaround time is also comparatively small. Table 15 and Table 16 show the results in tabular form.

\section{Evaluation of response time}

As a second performance metric, we consider the response time of the algorithm to incoming tasks. The response time is essentially the time during which the request is actually considered. In other words, we can say that the response time is directly dependent on the availability of resources. The availability of resources is dependent up on the scheduling of tasks. If the scheduling of tasks is performed properly, then the resources will naturally be free early or in advance of deadlines, the response times will be less in such cases.

By, comparing the response times obtained for our proposed heuristic approach with those obtained using the existing BATS and IDEA frameworks, we can see that our system's response time is almost $50 \%$ less. The response time comparisons for Cybershake and Epigenomics are presented in Figs. 8 and 9 respectively. The comparison is also shown in tabular form in Tables 17 and 18. We consider two parameters the response time and turnaround time compare the proposed heuristic approach with the existing BATS and IDEA

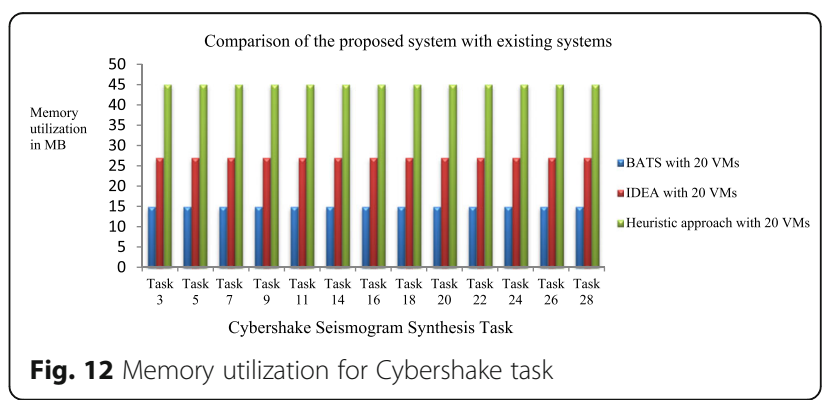


Comparison of the proposed system with existing systems

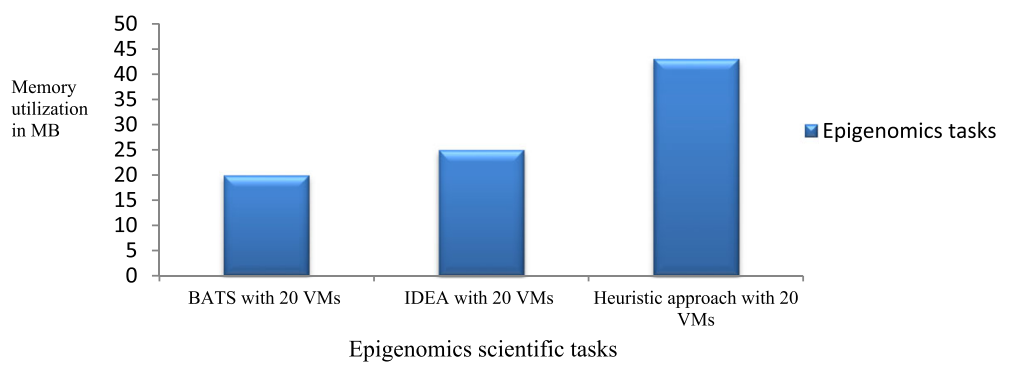

Fig. 13 Memory utilization for Epigenomics task

frameworks. Because we are evaluating these frameworks in a cloud computing environment, the response time is generally less effective.

On the other hand, we also evaluated our proposed heuristic approach to determine its resource performance compare it to those of the existing BATS and IDEA frameworks.

\section{Evaluation of CPU utilization}

Figures 10 and 11 show key comparison of resource utilization between the proposed heuristic approach and existing BATS and IDEA frameworks. The proper utilization of resources produces profits for cloud computing service providers. The experimental results shows that the proposed heuristic approach utilized the CPU resource more efficiently than the existing BATS framework.

\section{Evaluation of memory utilization}

Figures 12 and 13 show the second key comparison of resource utilization between the proposed heuristic approach and the existing BATS and IDEA frameworks. The experimental results shows that the proposed heuristic approach utilizes memory resources more efficiently than the existing BATS and IDEA frameworks.

\section{Evaluation of bandwidth utilization}

Bandwidth, an important resource, is not considered in most existing frameworks. We take bandwidth into account as a third important aspect of cloud computing data centers. We also compare our proposed heuristic approach with the existing BATS and IDEA frameworks. Figures 14 and 15 shows that our proposed heuristic approach utilizes bandwidth more efficiently than the existing BATS and IDEA frameworks.

\section{Conclusion}

In this study, we proposed heuristic algorithm that performs task scheduling and allocates resources efficiently in cloud computing environments. We use real Cybershake and Epigenomics scientific workflows as input tasks for the system. When we compare our proposed heuristic approach with the existing BATS and IDEA frameworks with respect to turnaround time and response time, we find that our approach gives improved results. On the other hand, from the viewpoint of resource utilization, the proposed heuristic approach efficiently allocates resources with high utility. We obtained the maximum utilization result for computing resources such as CPU, memory and bandwidth. Most existing systems consider only two resources, CPU and memory, in evaluating their performance the proposed system adds

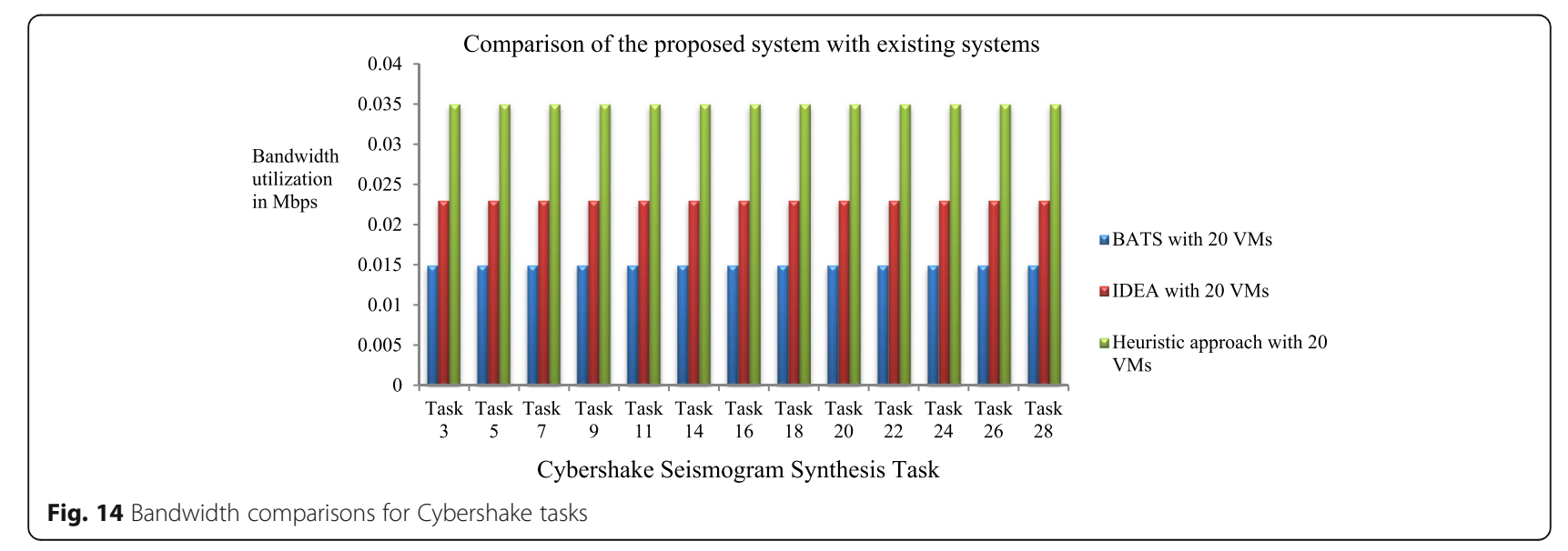


Comparison of the proposed system with existing systems

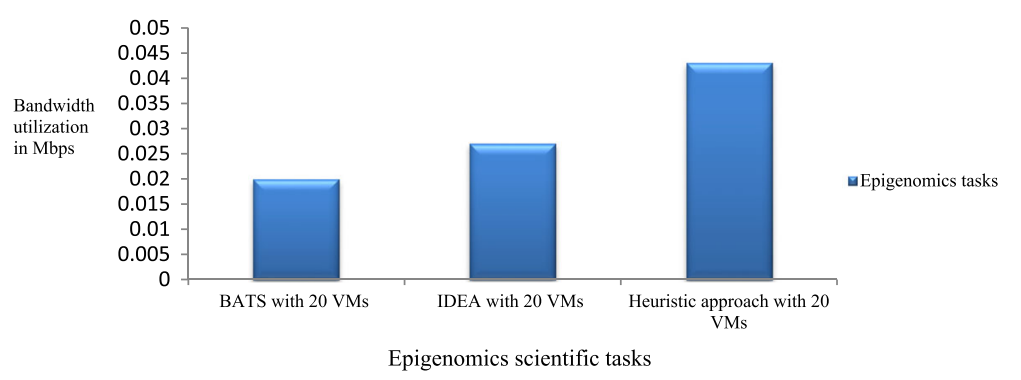

Fig. 15 Bandwidth comparisons for Epigenomics task

bandwidth as a resource. Future work will focus on more effective scheduling algorithms in which turnaround time and response time will be improved.

\section{Abbreviations}

AHP: Analytical hierarchy process; BATS: Bandwidth aware divisible task scheduling; FCFS: First come first served; GST: Green strain tensor; IDEA: Improved differential evolution algorithm; LEPT: Longest expected processing time; PSHA: Probabilistic seismic hazard analysis; SLA: Service level agreement

\section{Acknowledgments}

We would like to thank Mr. Amit Kolhe, the Managing Trustee of the Sanjivani College of Engineering, Kopargaon, India and the Principal of Thadomal Shahani Engineering College, Bandra (W), Mumbai, India, for providing the infrastructure needed to carry out the proposed research work.

\section{Funding}

Not applicable.

\section{Availability of data and materials}

The proposed heuristic approach is applied to real scientific tasks, i.e., Cybershake and Epigenomics, which are freely available for study and research. We provide the web site for these resources in the reference section under Reference-[22].

\section{Authors' contributions}

The work presented in this paper is based on Mahendra's Ph.D. research and thesis, which was supervised by Dr. SKS, who is a professor and contributed equally to this research. We implemented and performed the simulations presented in this study. Both of the authors read and approved the final manuscript.

\section{Competing interests}

The authors declare that they have no competing interests.

\section{Publisher's Note}

Springer Nature remains neutral with regard to jurisdictional claims in published maps and institutional affiliations.

\section{Author details}

'Department of Information Technology, Thadomal Shahani Engineering College, Bandra (W), University of Mumbai, Mumbai, MS, India. ${ }^{2}$ Lokmanya Tilak College of Engineering, Kopar Khairane, University of Mumbai, Mumbai, MS, India.

Received: 3 May 2017 Accepted: 26 January 2018

Published online: 08 February 2018

\section{References}

1. Gubbi J, Buyya R, Marusic S, Palaniswami M (2013) Internet of things (iot): a vision, architectural elements, and future directions. Futur Gener Comput Syst 29(7):1645-1660
2. Mezmaz M, Melab N, Kessaci Y, Lee YC, Talbi E-G, Zomaya AY, Tuyttens D (2011) A parallel bi-objective hybrid meta heuristic for energy-aware scheduling for cloud computing systems. J Parallel Distributed Computing 71(11):1497-1508

3. Armbrust M, Fox A, Griffith R, Joseph AD, Katz R, Konwinski A, Lee G, Patterson D, Rabkin A, Stoica l et al (2010) A view of cloud computing. Commun ACM 53(4):50-58

4. Tsai J-T, Fang J-C, Chou J-H (2013) Optimized task scheduling and resource allocation on cloud computing environment using improved differential evolution algorithm. Comput Oper Res 40(12):3045-3055

5. Maguluri ST, Srikant R (2014) Scheduling jobs with unknown duration in clouds. IEEE/ACM Trans Netw (TON) 22(6):1938-1951

6. Cheng C, Li J, Wang Y (2015) An energy-saving task scheduling strategy based on vacation queuing theory in cloud computing. Tsinghua Sci Technol 20(1):28-39

7. Lin W, Liang C, Wang JZ, Buyya R (2014) Bandwidth-aware divisible task scheduling for cloud computing. Software: Practice and Experience 44(2):163-174

8. Ergu D, Kou G, Peng Y, Shi Y, Shi Y (2013) The analytic hierarchy process: task scheduling and resource allocation in cloud computing environment. The Journal of Supercomputing. 64(3):835-848

9. Zhu X, Yang LT, Chen H, Wang J, Yin S, Liu X (2014) Real-time tasks oriented energy-aware scheduling in virtualized clouds. IEEE Transactions on Cloud Computing 2(2):168-180

10. Liu X, Zha Y, Yin Q, Peng Y, Qin L (2015) Scheduling parallel jobs with tentative runs and consolidation in the cloud. J Syst Softw 104:141-151

11. Shamsollah G, Othman M (2012) Priority based job scheduling algorithm in cloud computing. Procedia Engineering 50:778-785

12. Polverini M, Cianfrani A, Ren S, Vasilakos AV (2014) Thermal aware scheduling of batch jobs in geographically distributed data centers. IEEE Transactions on Cloud Computing 2(1):71-84

13. Rodriguez MA, Buyya R (2014) Deadline based resource provisioningand scheduling algorithm for scientific workows on clouds. IEEE Transactions on Cloud Computing 2(2):222-235

14. Keshk AE, El-Sisi AB, Tawfeek MA (2014) Cloud task scheduling for load balancing based on intelligent strategy. Int J Intell Syst Appl 6(5):25

15. Ghanbari S, Othman M, Leong WJ, Bakar MRA (2014) Multi-criteria based algorithm for scheduling divisible load. In: Proceedings of the first international conference on advanced data and information engineering (DaEng-2013), pp 547-554

16. Ghanbari S, Othman M, Bakar MRA, Leong WJ (2015) Priority-based divisible load scheduling using analytical hierarchy process. Appl Math Inf Sci 9(5):25-41

17. Goudarzi H, Ghasemazar M, Pedram M (2012) Sla-based optimization of power and migration cost in cloud computing. In Proceedings of the 2012 12th IEEE/ ACM International Symposium on Cluster, Cloud and Grid Computing (ccgrid 2012) (pp. 172-179). IEEE Computer Society

18. Radojevic B, Zagar M (2011) Analysis of issues with load balancing algorithms in hosted (cloud) environments. In: MIPRO, 2011 proceedings of the 34th international convention, pp 416-420

19. Ghanbari S, Othman M, Bakar MRA, Leong WJ (2016) Multi-objective method for divisible load scheduling in multi-level tree network. Futur Gener Comput Syst 54:132-143

20. Goswami S, Das A (2017) Optimization of workload scheduling in computational grid. In: Proceedings of the 5th international conference on Frontiers in intelligent computing: theory and applications, pp 417-424 
21. Juve G, Chervenak A, Deelman E, Bharathi S, Mehta G, Vahi K (2013) Characterizing and profiling scientific workflows. Futur Gener Comput Syst 29(3):682-692

22. Center SC (2014) Cybershake and Epigenomics scientific workflow. https://confluence.pegasus.isi.edu/display/pegasus/WorkflowGenerator. Accessed 1 Jan 2016

23. Handfield R, Walton SV, Sroufe R, Melnyk SA (2002) Applying environmental criteria to supplier assessment: a study in the application of the analytical hierarchy process. Eur J Oper Res 141(1):70-87

24. Del Acebo E, de-la Rosa JL (2008) Introducing bar systems: a class of swarm intelligence optimization algorithms. In: AISB convention communication, interaction and social intelligence, pp 18-23

25. Calheiros RN, Ranjan R, Beloglazov A, De Rose CA, Buyya R (2011) Cloudsim: a toolkit for modeling and simulation of cloud computing environments and evaluation of resource provisioning algorithms. Software: Practice and Experience 41(1):23-50

\section{Submit your manuscript to a SpringerOpen ${ }^{\odot}$ journal and benefit from:}

- Convenient online submission

- Rigorous peer review

- Open access: articles freely available online

- High visibility within the field

- Retaining the copyright to your article

Submit your next manuscript at $\gg$ springeropen.com 